\title{
Structure design and simulation of a new fire rescue equipment
}

\author{
Jianguo $\mathrm{LUO}^{1, \mathrm{a}}$, Zehao BU ${ }^{2, \mathrm{~b}}$ \\ ${ }^{1}$ Department of Mechanical and Electrical Engineering, North China Institute of Science and \\ Technology, East Suburb of Beijing, 101601, China \\ ${ }^{2}$ Department of Graduate School, North China Institute of Science and Technology, East Suburb of \\ Beijing, 101601, China \\ aemail: luojg_1598@126.com, bemail:buzehao@163.com
}

\section{Keywords: Structure Design; Simulation; High-rise Building Fire; Fire Rescue Equipment}

\begin{abstract}
With the increase of high-rise buildings, high-rise building fire accidents also tend to be normalization. High-rise building fire, particularly ultra-high-rise building fire, has become an unsolvable problem in life and workplace. The design of new embedded scalable fire rescue equipment will effectively improve the ability of high-rise firefighting and rescue, and also make some special design of the structure according to the special environment. Modeling the embedded fire rescue equipment through PTC Creo3.0 and studying the process of assembling and simulation to test whether motor function meets the design requirements and actual operational requirements or not.
\end{abstract}

\section{Introduction}

Since urbanization and economic development require more space, constructing high-rise building has become an effective means of saving space. Ultra-high-rise buildings in large urban agglomerations address the shortage of land, but bring the problem of fire prevention and control into a high level [1]. High-rise building firefighting faces big difficulties in water supply, gas flow which is influenced by the height, dangerous, climbing challenge, difficult to use vehicle, the radio communication signal attenuation serious, communicate blind spots and other issues [2]. High water tank to serve water, elevator evacuation are often used for high-rise building fire rescue [3]. Fire rescue mode is divided into self - help and help from others. In high-rise buildings, self-help firefighting equipment mainly include: indoor and outdoor fire hydrant system, automatic alarm system, automatic sprinkler system; helping from others mainly includes the equipment of fire helicopter, firefighting robots, fire vehicles, fire guns [4]. In recent year water bomber helicopter, fire robot, fire guns also get some breakthrough [5]. In June 2002, by the Ministry of Public Security Shanghai Fire Research Institute, Shanghai Jiao tong University and Shanghai Fire Bureau shared three units of 863 project "crawler, wheeled firefighting robot" successfully developed and passed the national acceptance. Firefighting robot, also known as self-propelled water - foam gun, is a combination of a variety of firefighting methods [6]. Analysis the applications of high-rise building fixed fire protection since 2000, the summary should be drawn as fixed fire fighting facilities within the high-rise building should be preferred firefighting equipment [7]. Embedded retractable fire rescue equipment is based on high-rise building fire, integrated with mechanical design and optimization, electrical system design and architecture, will achieve convenience, safety, reliability, intelligent of high-building fire rescue, then meeting the requirements of real-time monitoring and fire extinguishing.

\section{Overall Structure Design}

Embedded scalable high-rise building fire rescue device (Fig.2) consists of three parts: telescopic rails, fire extinguishing systems, ground remote central control system. Telescopic rails mounted horizontally in a high-rise building refuge floors, installed with extinguishing medium and scalable transport pipeline (Figure 1). Fire extinguishing system consists of control room, fire guns, fire 
monitoring devices, gun control system, fire monitoring transmission system, the inherent power. Remote central control system consists of ground fire live image display system, the gun remote control system, extinguishing medium transport control system. When fire occurs, it can manually or automatically extend outside the building, fire extinguishing system is fixed at the end of the telescopic rails. Fire monitoring device adjust to achieve three-dimensional direction, collecting real fire screen, the information is sent wirelessly to the control room, control room send instructions to the gun control system based on radio transmission, adjusted by the gun control system to achieve precisely firefighting. Single gun perpendicular and adjacent to at least cover the scope of work for more than half the distance of two layers of refuge, horizontal single gun could cover same range with the perpendicular single gun, the working scope depends on the size and level of fire extinguishing system and the number and length of guns.

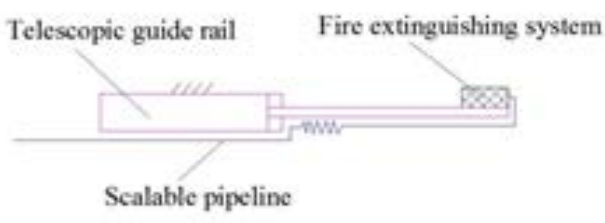

Figure 1: Telescopic program diagram

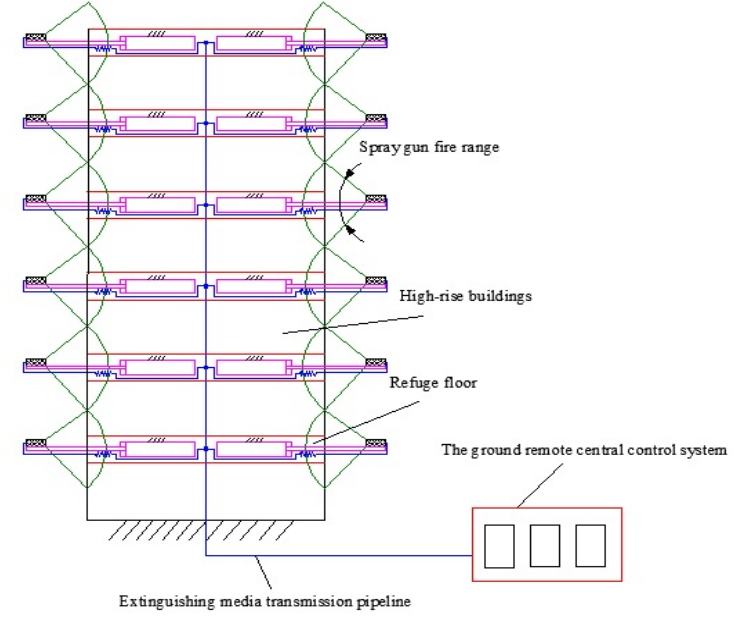

Figure 2: Overall Equipment Installation Diagram

\section{Telescopic cylinder design}

The largest travel requirement of retractable cylinder in design is $50 \mathrm{~m}$, initially using six telescopic cylinders, stroke of each stage $5 \mathrm{~m}$. During their work, it needs to extend and retract the cylinders, all levels cylinder stretch sufficient stroke in order, choose multi-stage double-acting telescopic cylinders. In the process of working, there is a large amount of compressed gas in the cylinder, after the cylinder fully extending, the cylinder will be under great bending stress, and the wall thickness of the cylinder must be reached certain requirements to ensure their normal working condition. The basic parameters of the multi-stage telescopic cylinders are in the table below:

Table 1: The main dimensions of the cylinder at all levels

\begin{tabular}{|c|c|c|c|c|c|c|}
\hline Attributes & 1st & 2nd & 3rd & 4th & 5th & 6th \\
\hline the inside diameter D/mm & 400 & 320 & 250 & 180 & 110 & 50 \\
\hline Wall thickness $\delta / \mathrm{mm}$ & 17 & 17 & 17 & 14 & 14 & 10 \\
\hline the outer diameter d/mm & 434 & 354 & 284 & 208 & 138 & 70 \\
\hline
\end{tabular}

Telescopic cylinder structure with the advantage of light, easy to drive, small moment of inertia and a small stretch of space make it easy to do stretch operation. In addition, during pneumatic drive, it can adapt to a wide temperature range, fire and explosion prevention and other features also make it adapt under fire, such structure can adapt to extreme conditions stretch process, and to achieve a high temperature support. Cylinder Head main has sealing and axial positioning two functions. Between the cylinder and the cylinder head, it needs to be closed to prevent leakage. O-ring seals can be fixed, and requires air intake and exhaust vents to ensure the normal operation of the cylinder. To ensure the trachea and the multi-stage telescopic cylinders synchronized 
telescopic movement, using spiral hose. Material is PU (polyurethane), the maximum working pressure is $0.5 \mathrm{MPa}$, Withstanding pressure is $1.5 \mathrm{MPa}$. The maximum working length $3000 \mathrm{~mm}$, screw outer diameter $\mathrm{D}=80 \mathrm{~mm}$, pipe diameter $\mathrm{d}=15 \mathrm{~mm}$. When the pipe and the cylinder extending, the temperature of the fire spot is very high, give the pipeline with a layer of fire, high temperature protective cover, or some surface coating with protective materials.

\section{Angle adjustable sprinkler head design}

The design is necessary to consider a range of distance, also can be stably adjusted to ensure accurately firefighting; secondly, it has to be adjustable in remote control, so wireless transmission design is required; finally, it should be installed on the head of mounted telescopic mechanism and it needs special design. Through analysis, considering the working characteristics of the gun, the working environment and working methods, using a stepper motor driven approach can achieve better remote control, while it has light structure. So, choose motor-driven manner simultaneously have some adaptive structural design (Figure 3).

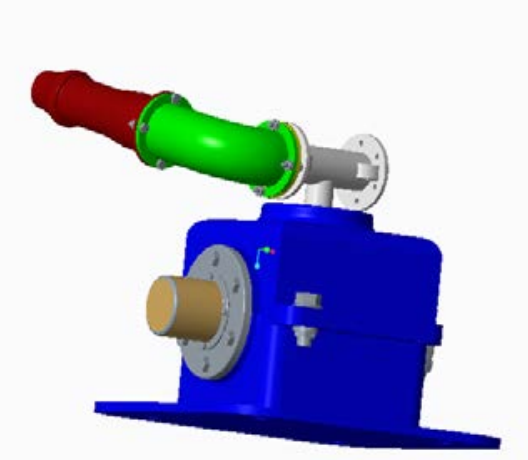

Figure 3: Adaptable two degrees of freedom motor gun

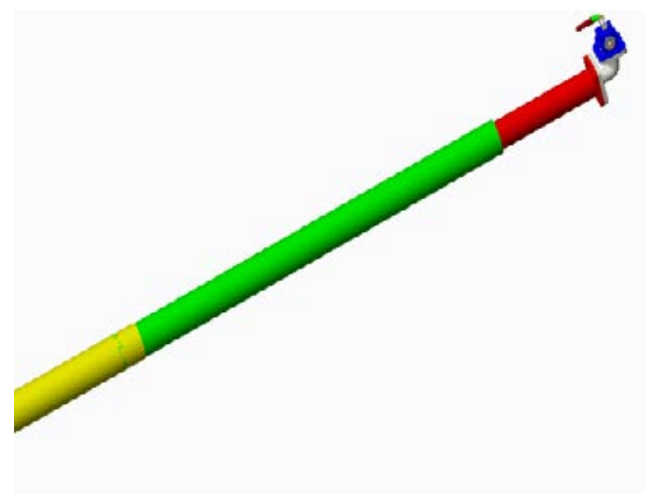

Figure 5: Cylinder assembly

\section{Embedded design and Modeling}

According to the relevant statements of China's "design specifications of high level residential mansions": "When the height of building is over than 100 meters, they should be set refuge floor (room)." Refuge layer is typically combined with the device layer, fire extinguishing systems and fire smoke isolation system. Cylinder assembly on the refuge layer disposed telescopic arm structure placed mosaic groove, in order to simulate the effect, installed the general room simulation model and human body models, model assembly are shown in Figure 4:

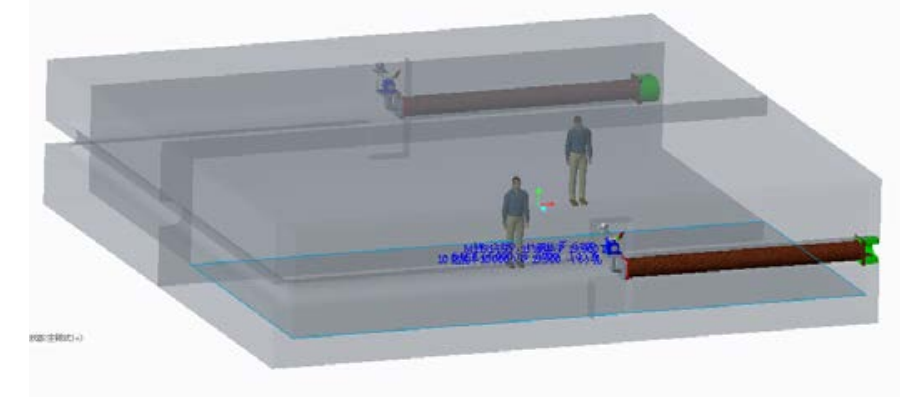

Figure 4: Embedded scalable fire rescue system

In Creo3.0 organization module, it can make action simulate and analysis of a mechanism device, in addition to viewing the bodies in action, with or without collision during body movement, also can get the position analysis, action analysis, dynamic analysis, static analysis and force balance analysis, to provide reference data for the improved design.

During assembly of cylinder(FIG. 5), pay attention to the reference interface, regardless of the definition of rotation of the motor cylinder or the definition of the amount of displacement in the 
direction of movement are based on the reference plane of zero, the rebuild also based on the zero value as the initial plane surface when assembly model.

\section{Simulation analysis}

Gun (Fig. 3) has rotational movement in the form of two degrees of freedom, in setting movement, as long as set its two rotate drive motors. Figure 6 is a rotational position of the drive motor in the Z-axis direction, the images of angular velocity and angular acceleration. Within the time range $0-20$ s, the angular displacement of the gun in the Z-axis direction is close to zero, there is no rotation at this position, angular velocity and angular acceleration is also zero; and within 20-30s, a larger rotation angle in $\mathrm{Z}$ axis at this time is to adjust the spray gun in a vertical direction, to rotate the gun to fire position, speed and acceleration throughout the adjustment process has reached a maximum value, the maximum speed is about $-156^{\circ} / \mathrm{min}$, maximum acceleration can reach $-580^{\circ} / \mathrm{min}$; during $30-40$ s, spray gun position remains unchanged in the process is to simulate gun firefighting process, just let the gun stayed for ten seconds to simulate the fire under control, fire rescue system can be embedded motion back during the time 45-60s, Z-axis slowly back to the initial position.

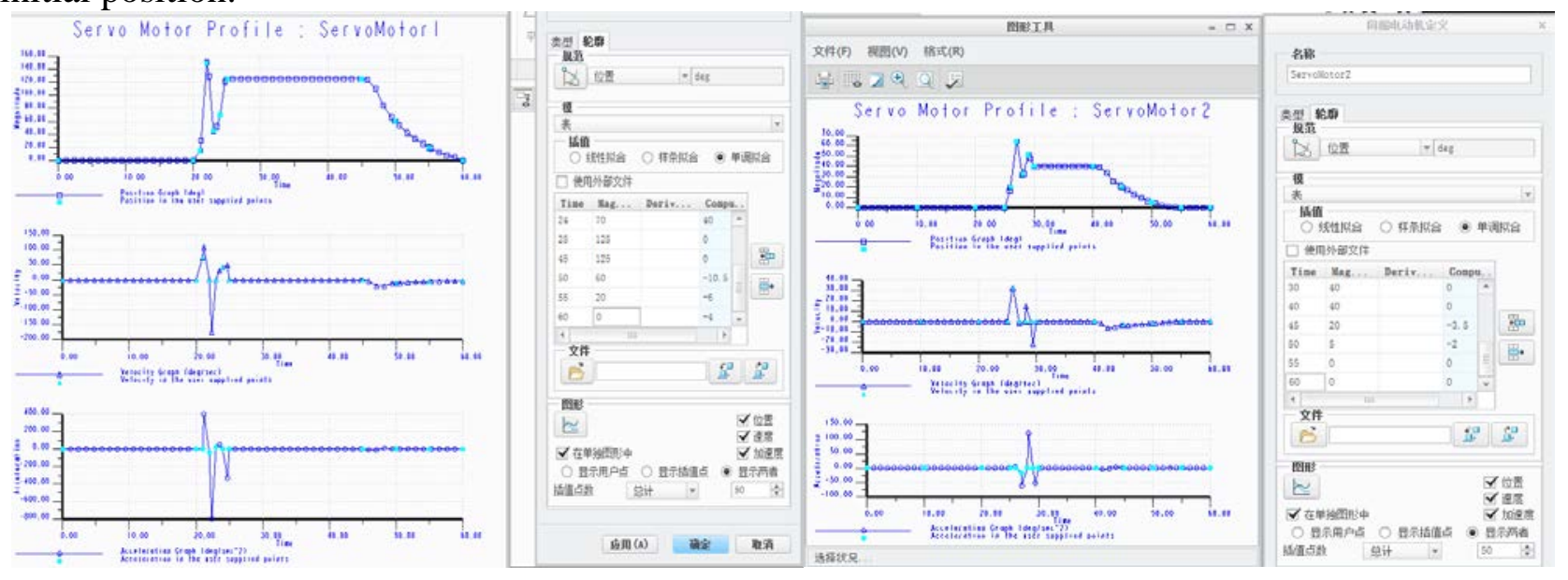

Figure 6: Z-axis drive motor motion parameters $\quad$ Figure 7: X-axis drive motor motion parameters

Set the X-axis motor movement parameter (Figure 7). Consider the convenience of control on the two rotation sequence control, the Z-axis motor is driven to rotate in the process, $\mathrm{X}$-axis motor does not rotate; ensure that the final adjustment process control gun can put out fire accurately. Just as showed in figure 7 , during the movement of the $\mathrm{X}$-axis drive parameter setting, also refer to the embedded scalable fire rescue system, there is no angle change during $0-20$ s, when the main cylinder is rotating and extending; after 20s, with the angular velocity and angular acceleration occurs, it rotates in the X-axis, in order to simulate the adjustment process, setting the gun cannot be located precisely in one adjustment process, in the 30s the adjustment is complete, the whole process, the maximum angular displacement occurs around 27s, the maximum angular displacement of $63^{\circ}$, the maximum angular acceleration occurred at about 26s, the maximum angular velocity of $80 \%$, the maximum angular acceleration occurs near the 28s, the maximum angular acceleration of about $100^{\circ}$ s; as description above in $30-40$ s gun position does not move, kept at $49^{\circ}$ position put out the fire, then with the recovery of the entire system, the gun is reset.

In the above description of the gun simulation, it is gun movement process in 20-30s; 30-40s is to simulate gun firefighting process. Extend and recovery movements of telescopic are set in 0-20s, 40-60s. Set driven process, since the simulation pneumatic telescopic pneumatic cylinder has six sections, so in order to simulate the valve twice commutation specific process, add five drivers to control each section of the cylinder extending and recovery process, as shown in Figure 9,10,11 for the first three drivers. The telescopic arm rotate out and the process of recovery are relatively 
simple; to simplify the motion simulation model, only add the rotation drive systems control pneumatic telescopic arm. Driven roll-out recycling movement shown in Figure 8.

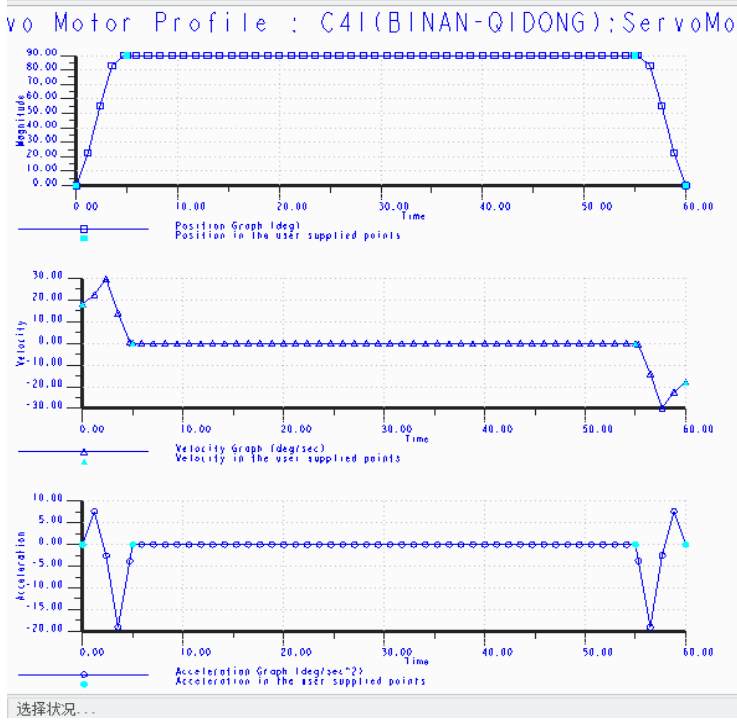

Figure 8: Rotate drive

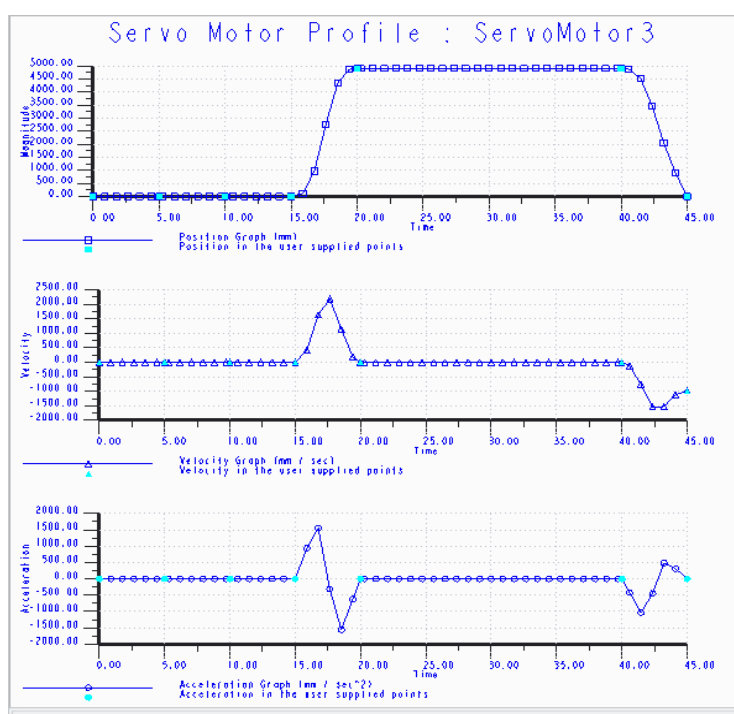

Figure 10: Cylinder drive 2

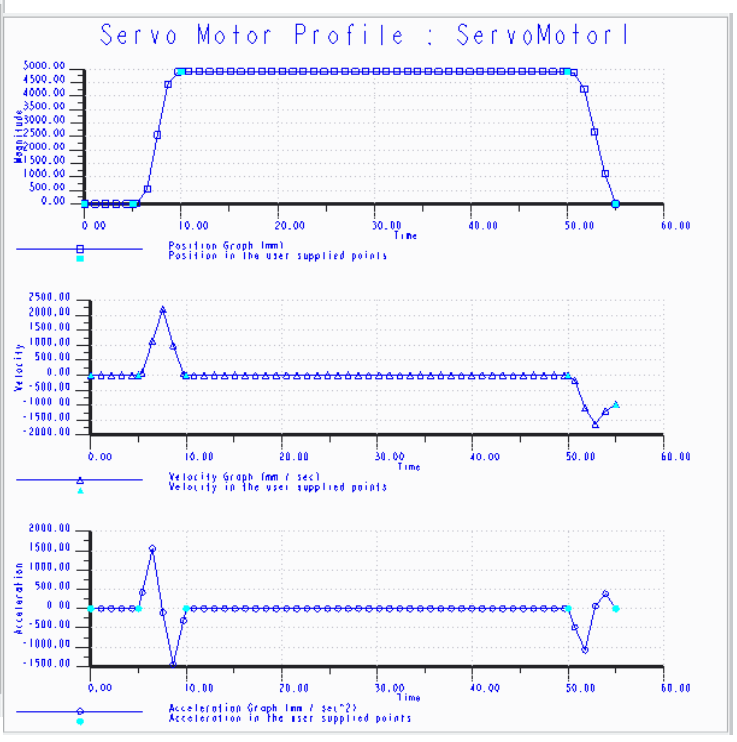

Figure 9: Cylinder drive 1

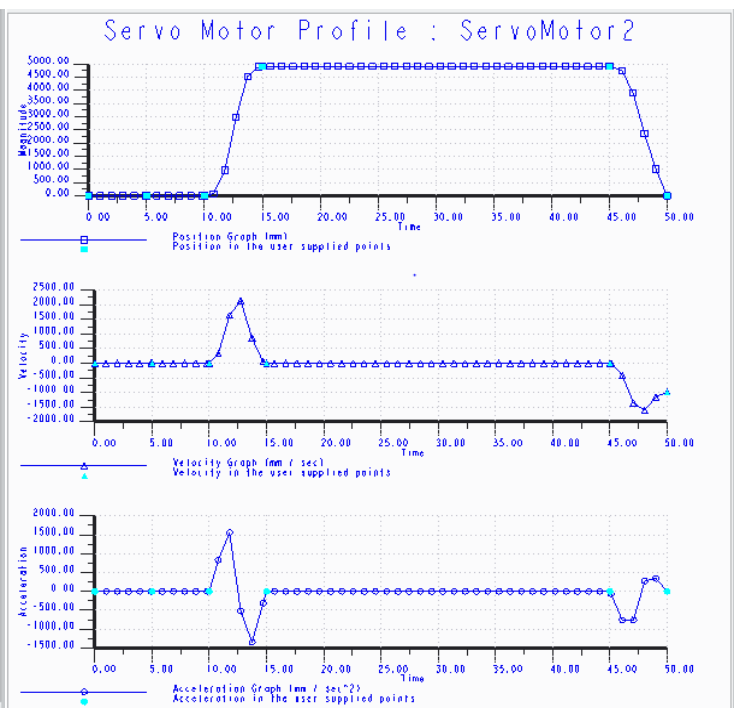

Figure 11: Cylinder drive 3

\section{Conclusion}

(1) Make system and structural design for the embedded scalable rescue equipment, modeling by using Creo3.0 software, use mechanism module to make dynamic simulate of the embedded scalable fire rescue equipment, installed in refuge layer, the stretch process of multi-stage cylinder, and the gun control movement.

(2) Compare and analysis the simulation data, the gun movement with two degrees of freedom and movement of the telescopic cylinder worked follow the basic form that has been set up, achieve the desired design effect.

(3) In the background of high-building firefighting and rescue, explain the working progress of embedded scalable fire rescue equipment, make dynamic modeling and simulation based on PTC Creo3.0 simulation software; providing a theoretical basis for further optimization of device and the actual operation, helping promote the use of the equipment, and have a positive meaning for the prevention and rescue of high-rise building fire. 


\section{Acknowledgement}

This research was financially supported by the Hebei Province Science and Technology Foundation (No.Z2015072, No.15275508) and NCIST Foundation (No. 3142015023).

\section{References}

[1] Y. Q. Luo, High-rise building fire safety analysis and countermeasures [J]. Fire Science and Technology .2012 (12)

[2] H. Q. Liu, H. D. Wei, Difficulties and Countermeasures of high-rise building fire fighting [J]. Police Force Academy, 2015 (08)

[3] L. Wang, K. Q. Kuang, High-rise building fire safety strategy case study. [J]. Building fire protection design $2012(02)$

[4] Y. Wang, High-rise building fire extinguishing countermeasures [J]. TECHNIQUE AND PRODUCTS INFORMATION .2010 (07)

[5] J. Liu, J. G. Cheng, Z. Yin, Y. Z. Shen, Fire fighting and rescue robot application of technical analysis. [J]. TECHNIQUE AND PRODUCTS INFORMATION .2010 (11)

[6] J. G. Liu, Y. C. Wang, B. Li, S. G. Ma. Disaster robot research status, key performance and prospects [J]. Mechanical Engineering. 2006 (12)

[7] Y. M. Zhang, L. B. Li, High-rise building fire fixed fire facilities Application [J]. Fire Science and Technology .2012 (04) 